\title{
ELABORAÇÃO DE PÃO FRANCÊS COM FARINHA ENRIQUECIDA DE SULFATO DE CÁLCIO
}

\author{
SHIZUKO KAJISHIMA * \\ MATILDE PUMAR ** \\ ROGÉRIO GERMANI ***
}

\begin{abstract}
Este trabalho teve como objetivo estudar os efeitos da adição de sulfato de cálcio nas características físicas e reológicas da farinha e na qualidade do pão francês. Foram utilizados dois níveis de cálcio: $50 \%(400 \mathrm{mg})$ e $100 \%$ (800 mg) da ingestão diária recomendada (IDR) para $100 \mathrm{~g}$ de pão. $\mathrm{Na}$ farinha foram avaliadas a cor, o teor de glúten, a farinografia, a alveografia e a extensigrafia. Analisou-se o volume específico e conceito global do pão francês elaborado. $O$ sulfato de cálcio melhorou a cor da farinha e sua estabilidade à mistura, diminuindo sua capacidade de absorção de água. Concluiu-se que é possível o enriquecimento do pão francês em níveis de até $100 \%$ da IDR de cálcio sem prejuízos significativos em sua qualidade.
\end{abstract}

PALAVRAS-CHAVE: PÃO FRANCÊS; FARINHA; SULFATO DE CÁLCIO.

\section{INTRODUÇÃO}

O beneficiamento de alimentos proporciona vantagens como a destruição de elementos indesejáveis e a melhoria de características sensoriais, porém afeta de modo especial o teor de vitaminas e minerais dos produtos (BARUFFALDI \& OLIVEIRA, 1998). As indústrias de alimentos demonstram particular interesse na reposição destas perdas e no valor nutricional dos produtos processados.

O pão, um dos alimentos mais difundidos, constitui-se numa das principais fontes calóricas da dieta do brasileiro (ABIP, 1999). Segundo o IBGE (1998), em 1995-1996 o consumo médio per capita de pão francês no Brasil foi de 18,399 kg/ano.

* Professora da Faculdade de Nutrição da Universidade Federal Fluminense (UFF), Rio de Janeiro, RJ. (e-mail: shinje@uol.com.br).

** Professora do Instituto de Nutrição, Universidade Estadual do Rio de Janeiro (UERJ), Rio de Janeiro, RJ.

*** Pesquisador da EMBRAPA - Agroindústria de Alimentos, Rio de Janeiro, RJ. 
A farinha de trigo é o elemento fundamental na indústria de panificação. $O$ pão francês, por tratar-se de formulação mais simples, não contém açúcar e tem pouca gordura, necessitando de farinha "forte" para sua produção (BENASSI \& WATANABE, 1997). Devido à presença de proteínas insolúveis (gliadina e glutenina), a farinha de trigo é capaz de formar rede de glúten e gerar massa forte e coesa, podendo reter gases em produtos fermentados, cuja cocção resulta em produto esponjoso (HOSENEY, 1991).

VANNUCCHI et al. (1990) consideraram a falta de cálcio como problema nutricional presente e potencial no Brasil. A deficiência de cálcio pode acarretar osteoporose em adultos e problemas ósseos em crianças, causando raquitismo, caracterizado pelo crescimento anormal dos ossos e deformidades em suas extremidades.

O Instituto Nacional de Saúde dos Estados Unidos sugeriu, para mulheres pós-menopausa, a ingestão de 1000 a $1500 \mathrm{mg}$ de cálcio, que no ano de 1985 era de somente $600 \mathrm{mg}$ (GREGER, 1987).

Não é nova a idéia de adicionar nutrientes em gêneros alimentícios. Em 1831 a adição de iodo em sal para prevenir o bócio foi recomendada. $O$ enriquecimento se aplica, em geral, ao alimento que contenha ou não vitaminas e minerais antes do beneficiamento e depende da legislação vigente no país. No Brasil, o Ministério da Saúde determina que os alimentos enriquecidos ou fortificados prontos para consumo devem conter em 100 mL no mínimo 15\% da Ingestão Diária Recomendada (IDR) e em $100 \mathrm{~g}$ no mínimo $30 \%$ da IDR para vitaminas e/ou minerais (BRASIL, 1998).

Nos EUA, em 1941, iniciou-se o enriquecimento de pão com cálcio, em quantidades equivalentes à sua perda durante o beneficiamento (DAVIS, 1981). O enriquecimento com vários nutrientes vem sendo recomendado, desde 1974, pela National Academy of Sciences/National Research Council (NAS/NRC, 1974).

A quantidade de sais de cálcio empregada no enriquecimento pode tornarse um problema, pois são necessárias $2,2 \mathrm{~g}$ a $3,5 \mathrm{~g}$ de sais para se alcançar a IDR deste mineral. Tais quantidades podem alterar a qualidade sensorial dos produtos como o aparecimento de sabor e odor de giz, sensação de areia na boca, mudança na cor e na textura (BARUFFALDI \& OLIVEIRA, 1998). Entre os sais de cálcio permitidos, o sulfato de cálcio apresentou melhor biodisponibilidade, conforme estudo experimental 
com animais usando pão de fôrma enriquecido com diferentes fontes de cálcio (RANHOTRA et al. 1997).

PARRISH et al. (1980), em seus estudos, recomendaram a adição de sulfato de cálcio na quantidade de 665 e $687 \mathrm{mg} / 100 \mathrm{~g}$ para farinha de trigo destinadas à produção de pão e bolo, respectivamente. RANUM (1980), em estudo de programa de enriquecimento de farinha de trigo, sugeriu $660 \mathrm{mg} / 100 \mathrm{~g}$ de sulfato de cálcio.

Segundo EL-DASH, CAMARGO \& DIAZ(1982) a presença de sulfato de cálcio na massa e no pão (50-300 ppm), aumenta a absorção de água em $2 \%$, com efeito benéfico na massa e no pão, aumentando seu volume. Tais autores também evidenciaram que o cálcio, na concentração de 1350 ppm, exerce efeito benéfico na fermentação.

Segundo GERMANI et al. (1997) a presença de sais de cálcio provoca efeito sobre o fortalecimento do glúten e atua ligeiramente como nutriente para o fermento. Outros autores afirmam que a quantidade de, aproximadamente, $300 \mathrm{mg}$ de cálcio em 453,6 g de pão não provoca efeito desfavorável na qualidade do produto (RANHOTRA et al., 1997).

Considerando o alto consumo de pão francês pela população brasileira este apresenta grande potencial para promover maior ingestão de cálcio.

Este trabalho teve como objetivo avaliar os efeitos ao cálcio nas propriedades da massa e do pão francês obtidos de farinha enriquecida com este mineral.

\section{MATERIAL E MÉTODOS}

Utilizou-se farinha de trigo comercial fornecida pelo Moinho Fluminense $\mathrm{S} / \mathrm{A}$ (Rio de Janeiro), na qual adicionou-se sulfato de cálcio $\left(\mathrm{CaSO}_{4} \cdot 2 \mathrm{H}_{2} \mathrm{O}\right)$, com teor de cálcio de $170 \mathrm{mg} / \mathrm{g}$. O sulfato de cálcio moído e tamisado foi acrescentado a cada $100 \mathrm{~g}$ de farinha, nas quantidades de $2,35 \mathrm{~g} \mathrm{e}$ $4,69 \mathrm{~g}$, correspondendo a $50 \%$ (400 mg) e 100\% (800 mg) da IDR de cálcio (BRASIL, 1998). A mistura foi devidamente homogeneizada em equipamento marca CHOPIN (Trippete \& Renauld, França), por 15 minutos (PARRISH et al., 1980).

Determinou-se a cor da farinha e da mistura com colorímetro KENT-JONES \& MARTINS (Flour color grade series 2), segundo o manual de instrução do fabricante (Henry Simon Limited, Stockport, Inglaterra). 
Os teores de glúten úmido e seco, bem como o índice de glúten foram determinados pelo método 38-12 da AACC (1995), utilizando-se aparelho GLUTOMATIC.

As características da mistura foram determinadas de acordo com o método 54-21 da AACC (1995) utilizando-se farinógrafo Brabender com cuba misturadora para $300 \mathrm{~g}$. Os parâmetros empregados para interpretar o farinograma foram: absorção de água (ABS), tempo de desenvolvimento da massa (TDM), estabilidade (EST), índice de tolerância à mistura (ITM) e valor do valorímetro (VV). Outras características de extensão foram determinadas pelo teste de alveografia, segundo método 54-30A da AACC (1995), utilizando-se alveógrafo CHOPIN, modelo 8120, com RCV-4 da mesma marca. Os parâmetros analisados envolveram: tenacidade ou sobrepressão máxima $(P)$, extensibilidade ou abscissa média de ruptura $(L)$, relação entre $P$ e $L$ e a força do glúten $(W)$. Estas características também foram determinadas pelo extensígrafo Brabender, segundo método 54-10 da AACC (1995), empregando-se os seguintes parâmetros para avaliação dos resultados: área $(A)$, resistência máxima à extensão $(R m)$, extensibilidade (E) e número proporcional (Rm/E).

A panificação baseou-se na descrição de EL-DASH, MAZZARI \& GERMANI (1994), mediante processo direto, utilizando-se masseira lenta. A formulação de pão francês foi estabelecida após ensaios prévios (Tabela 1).

\section{TABELA 1 - FORMULAÇÃO DE PÃO FRANCÊS}

\begin{tabular}{l|c}
\hline INGREDIENTES & QUANTIDADE \\
\hline & \\
Farinha padra o ou farinha enriquecida com sulfato de c/Ftcio & $3000 \mathrm{~g}$ \\
Sal & $60 \mathrm{~g}$ \\
Fermento biol gico fresco & $36 \mathrm{~g}$ \\
Gordura hidrogenada & $30 \mathrm{~g}$ \\
cido asc rbico & $150 \mathrm{ppm}$ \\
Condicionador de massa & $30 \mathrm{~g}$ \\
gua & $1750 \mathrm{~mL}$ \\
\end{tabular}


Para avaliação do pão foi utilizada média ponderal, sendo atribuídos pesos 60 para o volume específico e 10 para cada um dos atributos (cor da crosta, simetria, cor do miolo, grão e textura), que totalizaram ao final o máximo de 100 pontos. Este escore foi, então, convertido em conceito global: excelente (91-100), muito bom (81-90), bom (71-80), regular (6170) e deficiente (<60), conforme GERMANI \& BENASSI (1998).

O volume e peso dos pães foram medidos após o resfriamento dos mesmos, ou seja, 30-60 minutos após retirados do forno (GERMANI \& BENASSI, 1998). Determinou-se o volume dos pães pelo deslocamento de sementes de painço e o volume específico, dividindo-se o volume do pão $\left(\mathrm{cm}^{3}\right)$ pelo seu peso (g) (EL-DASH; CAMARGO \& DIAZ, 1982).

Os dados obtidos foram submetidos à análise de variância (ANOVA), com o procedimento GLM (General Linear Model), pacote estatístico SAS (Statistical Analysis System). Empregou-se o teste de Tukey, para comparação entre médias, ao nível de significância de $5 \%$ de probabilidade.

\section{RESULTADOS E DISCUSSÃO}

Verificou-se diferença significativa entre as amostras padrão e as adicionadas de cálcio com 50\% (400 mg) e 100\% (800 mg) da IDR, conforme a Tabela 2. A diminuição no valor da cor, que indica o aumento do branqueamento da farinha conforme o aumento do enriquecimento, também foi relatada por BARUFFALDI \& OLIVEIRA (1998).

\section{TABELA 2 - COR (KENT \& JONES) DAS FARINHAS DE TRIGO}

\begin{tabular}{|c|c|}
\hline AMOSTRAS & COR * \\
\hline Farinha padr ${ }^{a} 0$ & $4,5 \mathrm{a}$ \\
\hline Farinha com $50 \%$ da IDR de sulfato de $\mathrm{Ca}$ & $3,8 b$ \\
\hline Farinha com $100 \%$ da IDR de sulfato de Ca & $3,7 b$ \\
\hline 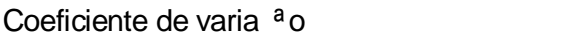 & 2,03 \\
\hline
\end{tabular}

* Médias seguidas por letras iguais, na vertical, não diferem significativamente $(p \leq 5 \%)$. 
Não houve diferença significativa entre as amostras em relação aos teores de glúten úmido, seco e índice de glúten (Tabela 3). Os valores de teor de glúten úmido foram considerados médios e o índice de glúten alto, conforme estudo realizado por GERMANI \& BENASSI (1998). Segundo POMERANZ (1988) e GERMANI et al. (1997) a presença de sais de cálcio fortalecem a formação de glúten e como conseqüência facilitam a sua extração.

\section{TABELA 3 - AVALIAÇÃO DO GLÚTEN DAS FARINHAS DE TRIGO}

\begin{tabular}{|c|c|c|c|}
\hline AMOSTRAS & $\begin{array}{c}\text { GL TEN } \\
\text { MIDO }^{*} \\
(\%)\end{array}$ & $\begin{array}{c}\text { GL TEN } \\
\text { SECO * } \\
(\%)\end{array}$ & $\begin{array}{l}\text { "NDICE DE } \\
\text { GL TEN * } \\
(\%)\end{array}$ \\
\hline Farinha padr $r^{\mathrm{a}} \mathrm{o}$ & $24,15 a$ & $8,1 \mathrm{a}$ & $98,0 \mathrm{a}$ \\
\hline Farinha com $50 \%$ da IDR de sulfato de Ca & $21,90 \mathrm{a}$ & $8,5 a$ & $99,5 \mathrm{a}$ \\
\hline Farinha com $100 \%$ da IDR de sulfato de Ca & $22,05 a$ & $8,2 a$ & $98,0 a$ \\
\hline Coeficiente de varia $\stackrel{a}{0}$ & 2,65 & 9,90 & 0,92 \\
\hline
\end{tabular}

* Médias seguidas por letras iguais, na vertical, não diferem significativamente ( $p \leq 5 \%)$.

A adição de diferentes níveis de cálcio exerceu efeito sobre as características de mistura das massas, determinadas pelo farinógrafo (Tabela 4). A absorção apresentou diferença significativa entre a farinha de trigo padrão e aquelas em que foi adicionado o cálcio, decrescendo conforme se aumentou a quantidade do nutriente. Não houve diferenças significativas quanto aos parâmetros tempo de desenvolvimento da massa (TDM) e valor do valorímetro (VV). Observou-se aumento na estabilidade da farinha com $50 \%$ da IDR de sulfato de cálcio seguido por decréscimo para a farinha com $100 \%$. Atribui-se o acréscimo à capacidade de fortalecimento do glúten causado por sais de cálcio (GERMANI et al, 1997). Já o decréscimo observado pode ter sido causado pela diluição do sulfato de cálcio no glúten.

Os resultados do teste de alveografia não evidenciaram diferença significativa nos parâmetros estudados (Tabela 5).

As características de extensão das massas (Tabela 6) não apresentaram diferença significativa na maioria dos parâmetros. Os melhores resultados referem-se à farinha com adição de 50\% da IDR de sulfato de cálcio, cujos valores dos parâmetros obtidos podem ser comparados aos do 
estudo realizado por GUTKOSKI et al. (1997). Na adição acima deste nível há decréscimo em todos os parâmetros. Tal fato confirma que a presença excessiva de sais de cálcio altera as propriedades reológicas da massa, embora as características da farinha para produção de pão francês sejam mantidas (GERMANI et al., 1993).

TABELA 4 - FARINOGRAFIA DAS FARINHAS DE TRIGO

\begin{tabular}{l|c|c|c|c|c}
\hline \multicolumn{1}{c|}{ AMOSTRAS } & ABS (\%) & TDM (min) & EST (min) & ITM (UF) & VV \\
\hline Farinha padr $\mathrm{a}$ o & $58,7 \mathrm{a}^{*}$ & $15,2 \mathrm{a}$ & $14,0 \mathrm{c}$ & $22,5 \mathrm{a}$ & $89,5 \mathrm{a}$ \\
\hline $\begin{array}{l}\text { Farinha com 50\% da IDR de } \\
\text { sulfato de Ca }\end{array}$ & $57,9 \mathrm{~b}$ & $15,7 \mathrm{a}$ & $24,1 \mathrm{a}$ & $30,0 \mathrm{a}$ & $89,5 \mathrm{a}$ \\
\hline $\begin{array}{l}\text { Farinha com 100\% da IDR de } \\
\text { sulfato de Ca }\end{array}$ & $57,2 \mathrm{~b}$ & $15,2 \mathrm{a}$ & $19,5 \mathrm{~b}$ & $32,5 \mathrm{a}$ & $89,5 \mathrm{a}$ \\
\hline \begin{tabular}{l} 
Coeficiente de varia a 0 \\
\hline
\end{tabular} & 0,29 & 2,29 & 3,40 & 10,18 & 0,79 \\
\hline
\end{tabular}

ABS = absorção de água; TDM = tempo de desenvolvimento da massa; EST = estabilidade; ITM = índice de tolerância da massa; UF = unidade farinográfica; VV = valor do valorímetro. * Médias seguidas por letras iguais, na vertical, não diferem significativamente $(p \leq 5 \%)$.

TABELA 5 - ALVEOGRAFIA DAS FARINHAS DE TRIGO

\begin{tabular}{|c|c|c|c|c|}
\hline AMOSTRAS & $\begin{array}{c}\mathbf{P} \\
\left(\mathrm{mmH}_{2} \mathrm{O}\right)\end{array}$ & $\begin{array}{c}\mathrm{L} \\
(\mathrm{mm})\end{array}$ & $\begin{array}{c}\mathrm{P} / \mathrm{L} \\
\left(\mathrm{mm} / \mathrm{mmH}_{2} \mathrm{O}\right)\end{array}$ & $\begin{array}{c}W \\
\left(10^{-4} \mathrm{~J}\right)\end{array}$ \\
\hline Farinha padri 0 & $129 a^{*}$ & $38 a$ & $3,45 a$ & $250 a$ \\
\hline $\begin{array}{l}\text { Farinha com } 50 \% \text { da IDR de sulfato de } \\
\mathrm{Ca}\end{array}$ & $125 a$ & $47 a$ & $2,72 a$ & $280 a$ \\
\hline $\begin{array}{l}\text { Farinha com } 100 \% \text { da IDR de sulfato } \\
\text { de } \mathrm{Ca}\end{array}$ & $128 a$ & $38 a$ & $3,47 a$ & $250 a$ \\
\hline Coeficiente de varia ${ }^{a} 0$ & 0,80 & 9,51 & 10,70 & 6,06 \\
\hline
\end{tabular}

$\mathrm{P}=$ tenacidade $\mathrm{L}=$ extensibilidade; $\mathrm{P} / \mathrm{L}=$ relação entre $\mathrm{P}$ e $\mathrm{L} ; \mathrm{W}=$ força do glúten.

* Médias seguidas por letras iguais, na vertical, não diferem significativamente ( $p \leq 5 \%)$. 
Não houve diferença significativa entre as amostras quanto ao volume específico, embora os pães elaborados com farinha enriquecida com cálcio tenham apresentado tendência em diminui-lo (Tabela 7). De acordo com EL-DASH, CAMARGO \& DIAS (1982) e GERMANI et al. (1997) a presença de sulfato de cálcio aumenta o volume do pão, devido ao efeito benéfico na fermentação. Entretanto, a concentração utilizada neste trabalho foi acima da empregada pelos autores citados.

\section{TABELA 6 - EXTENSIGRAFIA DAS FARINHAS DE TRIGO EM 135 MINUTOS DE DESCANSO}

\begin{tabular}{|c|c|c|c|c|}
\hline AMOSTRAS & 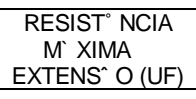 & $\begin{array}{l}\text { EXTENSIBILIDADE } \\
(\mathrm{mm})\end{array}$ & $\begin{array}{l}\mathrm{N}^{\circ} \text { PROPORCIONAL } \\
(\mathrm{Rm} / \mathrm{E})\end{array}$ & $\begin{array}{l}\text { REA } \\
\left(\mathrm{cm}^{2}\right)\end{array}$ \\
\hline Farinha padr ${ }^{\underline{a}} 0$ & $849 a^{*}$ & $74 a$ & $11,5 a$ & $72,5 a$ \\
\hline $\begin{array}{l}\text { Farinha com } 50 \% \text { da } \\
\text { IDR de sulfato de } \mathrm{Ca}\end{array}$ & $897 a$ & $81 a$ & $11,5 \mathrm{a}$ & $81,7 a$ \\
\hline $\begin{array}{l}\text { Farinha com } 100 \% \\
\text { da IDR de sulfato de } \\
\mathrm{Ca}\end{array}$ & $812 a$ & $68 a$ & $11,9 a$ & $61,8 a$ \\
\hline $\begin{array}{l}\text { Coeficiente de } \\
\text { varia }{ }^{\stackrel{a}{0}} 0\end{array}$ & 5,80 & 7,55 & 11,01 & 7,72 \\
\hline
\end{tabular}

UF= unidade farinográfica.

* Médias seguidas por letras iguais, na vertical, não diferem significativamente ( $p \leq 5 \%)$.

\section{TABELA 7 - VOLUME ESPECÍFICO E CONCEITO GLOBAL DO PÃO FRANCÊS PADRÃO E ELABORADOS COM AS FARINHAS DE TRIGO}

\begin{tabular}{|c|c|c|}
\hline $\mathrm{P}^{\wedge} \mathrm{OFRANC}^{\circ} \mathrm{S}$ & $\begin{array}{c}\text { VOLUME ESPEC'FICO* } \\
\left(\mathrm{cm}^{3} / \mathrm{g}\right)\end{array}$ & $\begin{array}{l}\text { CONCEITO } \\
\text { GLOBAL }\end{array}$ \\
\hline Farinha padr ${ }^{\mathrm{a}} \mathrm{o}$ & $7,64 \mathrm{a}$ & Muito bom \\
\hline Farinha com $50 \%$ da IDR de sulfato de $\mathrm{Ca}$ & $7,43 a$ & Muito bom \\
\hline Farinha com $100 \%$ da IDR de sulfato de Ca & $6,72 \mathrm{a}$ & Bom \\
\hline Coeficiente de varia $\stackrel{0}{0}$ & 6,19 & \\
\hline
\end{tabular}

* Médias seguidas por letras iguais, na vertical, não diferem significativamente ( $p \leq 5 \%)$. 
O conceito global não apresentou alteração significativa quanto à cor da crosta, simetria, cor do miolo, grão e textura. Entretanto, a adição de $100 \%$ da IDR de cálcio, ainda que em níveis aceitáveis, apresentou leve diminuição da quebra e leve opacidade na cor da crosta. Conforme estudos relatados por BARUFFALDI \& OLIVEIRA (1998) os produtos enriquecidos com este mineral apresentaram-se esbranquiçados.

\title{
4 CONCLUSÃO
}

Os dados experimentais do presente trabalho mostraram que a adição de sulfato de cálcio promoveu branqueamento da farinha de trigo. Sua adição, tanto em nível de 50\% (400 mg) quanto de 100\% (800 mg) da IDR, diminuiu a capacidade de absorção da farinha e melhorou a estabilidade da massa à mistura. Já o teor de glúten e os parâmetros de extensão avaliados, tanto no alveógrafo como no extensígrafo, não se mostraram significativamente diferentes $(p \leq 5 \%)$.

A avaliação da qualidade do pão francês enriquecido com cálcio mostrou que, embora o volume específico não tenha sido significativamente alterado, a adição da maior dosagem (100\% da IDR) causou leve diminuição em suas características externas.

Pode-se concluir que é possível o enriquecimento de pão francês em níveis de até $100 \%$ da IDR de cálcio sem prejuízos significativos em sua qualidade.

\begin{abstract}
FRENCH BREAD ELABORATION WITH CALCIUM SULFATE ENRICHED FLOUR

The objective of this work was to study the effect of calcium sulfate addition on the flour physical and rheological characteristics and on the French bread quality. Two levels of calcium were used: $50 \%(400 \mathrm{mg})$ and $100 \%(800 \mathrm{mg})$ of recommended dietary allowance (RDA) for $100 \mathrm{~g}$ of bread. Color, gluten content, farinograph and extensigraph were evaluated on the flour. The specific volume and global score on the bread were analyzed. Calcium sulfate improved the flour color and its mixing stability, decreasing the water absorption capacity. It was concluded that the enrichment of French bread at levels of until $100 \%$ of calcium RDA, without significant prejudices in its quality.
\end{abstract}

KEY-WORDS: FRENCH BREAD; FLOUR; CALCIUM SULFATE. 


\section{REFERÊNCIAS}

1 AACC. American Association of Cereal Chemists. Approved methods of the AACC. $9^{\text {th }}$ ed. St. Paul, 1995. $2 \mathrm{v}$.

2 ABIP. Associação Brasileira da Indústria de Panificação. O pão francês alimenta e pode ser usado em dietas. Disponível em: <http://www.abip.org.br/padaria/pt.htm>. Acesso em 12 out. 1999.

3 BENASSI, V.T.; WATANABE, E. Fundamento da tecnologia de panificação. Rio de Janeiro: EMBRAPA-CTAA, 1997. $60 \mathrm{p}$

4 BRASIL. Ministério da Saúde. Portaria n. 31 de 13 de jan. de 1998. Aprova regulamento técnico para fixação de identidade e qualidade de alimentos adicionados de nutrientes essenciais. Diário Oficial [da] República Federativa do Brasil, Brasília, 30 mar. 1998. p.6.

5 BRASIL. Ministério da Saúde. Portaria n. 33 de 13 de jan. de 1998. Ingestão Diária recomendada (IDR) de vitaminas, minerais e proteínas. Diário Oficial [da] República Federativa do Brasil, Brasília, 13 de jan. 1998. p. 2.

6 BARUFFALDI, R.; OLIVEIRA, M.N. Fundamentos de tecnologia de alimentos. São Paulo: Atheneu, 1998. v.3.

7 DAVIS, D. R. Wheat and nutrition. Nutrition Today, v. 16, n. 1, p. 16-21, Jul./Aug.,1981.

8 EL-DASH, A.A.; CAMARGO, C.O.; DIAZ, N.M. Fundamentos da tecnologia de panificação. São Paulo: Secretaria da Indústria, Comércio e Tecnologia, 1982. p. 1-243.

9 EL-DASH, A.; MAZZARI, M.R.; GERMANI, R. Tecnologia de farinhas mistas. Brasília: EMBRAPA-CTAA, 1994. v. 1, p. 42-88

10 GERMANI, R.; BENASSI, V.T.; CARVALHO, J.L.V.; TORREZAN, B.; CAMPOS, J.E.; MAZZARI, M.R. Curso para laboratoristas da indústria moageira de trigo. Rio de Janeiro: EMBRAPA/ MAARA/CTAA, 1993. 65 p. (Apostila).

11 GERMANI, R.; BENASSI, V.T.; CARVALHO, J.L.; WATANABE, E.; CAMPOS, J. E.; CARVALHO, C.W.P. Curso de controle de qualidade tecnológica do grão e da farinha de trigo. Rio de 
Janeiro: EMBRAPA-CTAA, 1997. 60 p. (Apostila).

12 GERMANI, R.; BENASSI, V.T. Misturas de farinhas de trigo: efeito na elaboração de pão francês e de fôrma. In: CONGRESSO BRASILEIRO DE CIÊNCIA E TECNOLOGIA DE ALIMENTOS, 16., Rio de Janeiro, 1998. Anais... Rio de Janeiro: SBCTA, 1998. v. 3, p. 1887-1889,

13 GREGER, J. L. Mineral bioavailability: new concepts. Nutrition Today, v. 22, p. 4-9, July/Aug., 1987.

14 GUTKOSKI, L.C.; PAVANELLI, A.P.; MIRANDA, M.Z.; CHANG, Y.K. Efeito de melhoradores nas propriedade reológicas e panificação da massa de farinha de trigo. Ciência e Tecnologia de Alimento, v.17, n.1, p. 11-16, jan-abr. 1997.

15 HOSENEY, R.C. Princípios de ciencia y tecnologia de los cereales, Zaragoza: Acribia, 1991. $321 \mathrm{p}$.

16 IBGE. Instituto Brasileiro de Geografia e Estatística. Pesquisa de orçamento familiares 1995-1996: consumo alimentar domiciliar per capita. Rio de Janeiro: IBGE, 1998. v. 2.

17 NATIONAL ACADEMY OF SCIENCES. National Research Council. Proposed fortification policy for cereal-grain products. Washington, DC, 1974. $36 \mathrm{p}$.

18 PARRISH, D.B.; EUSTACE, W.D.; PONTE, J.G.; HEROD, L. Distribution of vitamin $A$ in fortified flours and effect of processing, simulated shipping and effect of processing, simulated shipping and storage. Cereal Chemistry, v.54, n. 4, p. 284-287, 1980.

19 POMERANZ, Y. Wheat chemistry and technology. St. Paul, Minnesota, USA: American Association of Cereal Chemistry, 1988. v. 2.

20 RANHOTRA, G.S.; GELROTH, J.A.; LEINEN, S.D.; SCHNELLER, F.E. Bioavailability of calcium in breads fortified with different calcium sources. Cereal Chemists, v. 74, n. 4, p. 361-363, 1997.

21 RANUM, P.M. Note on levels of nutrients to add under expanded wheat flour fortification/enrichment programs. Cereal Chemistry, 
v.57, n.1, p.70-72, 1980.

22 VANNUCCHI, H.; MENEZES, E.W.; CAMPANARA, A.O.; LAJOLO, F.M. Cadernos de nutrição. Sociedade Brasileira de Alimentação e Nutrição, v. 2, p. 101-102, 1990. 